\title{
Cubic Dresselhaus Spin-Orbit Coupling in 2D Electron Quantum Dots
}

\author{
Jacob J. Krich and Bertrand I. Halperin \\ Physics Department, Harvard University, Cambridge, MA 02138
}

(Dated: October 7, 2018)

\begin{abstract}
We study effects of the oft-neglected cubic Dresselhaus spin-orbit coupling (i.e., $\propto p^{3}$ ) in GaAs/AlGaAs quantum dots. Using a semiclassical billiard model, we estimate the magnitude of the spin-orbit induced avoided crossings in a closed quantum dot in a Zeeman field. Using these results, together with previous analyses based on random matrix theory, we calculate corresponding effects on the conductance through an open quantum dot. Combining our results with an experiment on conductance through an $8 \mu \mathrm{m}^{2}$ quantum dot [D. M. Zumbühl et al., Phys. Rev. B 72, 081305 (2005)] suggests that 1) the GaAs Dresselhaus coupling constant, $\gamma$, is approximately 9 $\mathrm{eV} \AA^{3}$, significantly less than the commonly cited value of $27.5 \mathrm{eV} \AA^{3}$ and 2) the majority of the spin-flip component of spin-orbit coupling can come from the cubic Dresselhaus term.

PACS numbers: 73.21.La,73.23.-b,71.70.-d,05.45.Mt,73.63.Kv,72.20.My,72.20.-i
\end{abstract}

Control over electron spin in semiconductors has promise for quantum computing and spintronics. In such applications, it is essential to understand how the transport of an electron through a circuit affects its spin; i.e., we must understand spin-orbit coupling. In technologically important III/V semiconductor heterostructures, spin-orbit coupling originates in the asymmetry of the confining potential (called the Rashba term), which can be controlled by gates, and in bulk inversion asymmetry of the crystal lattice (called the Dresselhaus term). In quasi-2D systems, the Dresselhaus term has two components, one linear in the electron momentum and the other cubic. The cubic Dresselhaus term is usually neglected, as it is generally smaller than the linear contribution. Datta and Das proposed a spin-field-effect transistor (SFET) for quasi-1D ballistic wires with Rashba coupling [1]. Schliemann, Egues, and Loss proposed an SFET that can operate in diffusive quasi-2D systems based on tuning the Rashba and linear Dresselhaus terms to be equal in strength, which produces long spin lifetimes, neglecting the cubic Dresselhaus term [2]. The strengths of the spin-orbit terms are difficult to measure independently, but a full understanding of their strengths is crucial to making such devices. Additionally, in confined systems such as quantum dots, some effects of the linear spin-orbit terms are suppressed [3], and it is important to know the magnitude of the cubic Dresselhaus contribution, which could limit or even prevent the functioning of spintronic devices.

We characterize the strength of the cubic Dresselhaus term in a confined system by its effect on avoided crossings in an in-plane magnetic field $\mathbf{B}_{\|}$that couples only to the electron spin. With no spin-orbit coupling, each eigenstate can be written as a product of orbital state $|\alpha\rangle$ and spin quantized along $\mathbf{B}_{\|}$. Eigenstates $|\alpha \uparrow\rangle$ and $|\beta \downarrow\rangle$ become degenerate when $\epsilon_{\alpha}-\epsilon_{\beta}=E_{\mathrm{Z}}$, where $\epsilon_{\alpha, \beta}$ are the orbital energies and $E_{\mathrm{Z}}$ is the Zeeman energy [21], but spin-orbit coupling leads to avoided crossings.

In the first half of this Letter, we estimate the cubic
Dresselhaus contribution to the avoided crossings, which can be larger than the linear terms' contribution since the latter are suppressed for small $\mathbf{B}_{\|}[3]$. In the second half of this Letter, we relate these avoided crossings in closed quantum dots to the mean and variance of the conductance when the quantum dot is connected to ideal leads. We compare these predictions to the results of Zumbühl et al. on transport through an $8 \mu \mathrm{m}^{2}$ quantum dot [4, 5] and find that agreement is possible only if the cubic Dresselhaus coupling constant in GaAs, $\gamma$, is considerably less than the frequently cited value of $27.5 \mathrm{eV} \AA^{3}$ [6, 7] from $\mathbf{k} \cdot \mathbf{p}$ theory. A smaller value of $\gamma$ has also been suggested by experiments [8, 9] and band structure studies [10, 11, 12]. Even with this smaller value of $\gamma$, we find that the cubic Dresselhaus term is the dominant spinflip mechanism in the sample considered. Both of these results are significant for spintronic devices.

We consider conduction electrons in a 2D electron system grown on a (001) surface of a III/V semiconductor confined to a small area by a potential $V(\mathbf{r})$. We use an effective Hamiltonian

$$
\begin{aligned}
\mathcal{H}=\frac{\left(\mathbf{p}-\mathbf{A}_{\mathrm{so}}\right)^{2}}{2 m} & +\frac{\gamma}{2 \hbar^{3}}\left(p_{2}^{2}-p_{1}^{2}\right)(\mathbf{p} \times \boldsymbol{\sigma}) \cdot \hat{\mathbf{e}}_{3} \\
& +V(\mathbf{r})+\frac{1}{2} g \mu_{B} \mathbf{B} \cdot \boldsymbol{\sigma}
\end{aligned}
$$

where $\mathbf{p}=\mathbf{P}-e \mathbf{A} / c, \mathbf{P}$ is the canonical momentum, $\mathbf{A}$ is the vector potential from the perpendicular magnetic field, $\boldsymbol{\sigma}$ is the vector of Pauli matrices, $m$ is the effective mass, $\mathbf{A}_{\text {so }}=\hat{\mathbf{e}}_{1} \hbar \sigma_{2} / 2 \lambda_{1}-\hat{\mathbf{e}}_{2} \hbar \sigma_{1} / 2 \lambda_{2}$ is the effective spinorbit vector potential, which contains both the Rashba and linear Dresselhaus spin-orbit terms, and $\lambda_{1,2}$ are the (linear) spin-orbit lengths [13, 14]. We choose a coordinate system with axes $\hat{\mathbf{e}}_{1}=[110], \hat{\mathbf{e}}_{2}=[1 \overline{1} 0]$, and $\hat{\mathbf{e}}_{3}=[00 \overline{1}]$. The second term is the cubic Dresselhaus term.

In a system of linear size $L$, the linear spin-orbit terms can be gauged away to first order in $L / \lambda$ by the unitary transformation $\mathcal{H} \rightarrow U \mathcal{H} U^{\dagger} \equiv \mathcal{H}^{\prime}$ where $U=\exp (i \mathbf{r}$. 
$\mathbf{A}_{\text {so }}$ [13]. Expanding to leading order in $L / \lambda$,

$$
\begin{aligned}
\mathcal{H}^{\prime} & =\frac{1}{2 m}\left(\mathbf{p}-\mathbf{a}_{\perp}-\mathbf{a}_{\|}\right)^{2}+b^{\mathrm{Z}}+b_{\perp}^{\mathrm{Z}} \\
& +\frac{\gamma}{2 \hbar^{3}}\left(p_{2}^{2}-p_{1}^{2}\right)(\mathbf{p} \times \boldsymbol{\sigma}) \cdot \hat{\mathbf{e}}_{3}+V(\mathbf{r}),
\end{aligned}
$$

where $\mathbf{a}_{\perp}=\frac{\hbar \sigma_{3}}{4 \lambda_{1} \lambda_{2}}\left[\hat{\mathbf{e}}_{3} \times \mathbf{r}\right], \mathbf{a}_{\|}=\frac{\hbar}{6 \lambda_{1} \lambda_{2}}\left(x_{1} \sigma_{1} / \lambda_{1}+\right.$ $\left.x_{2} \sigma_{2} / \lambda_{2}\right)\left[\hat{\mathbf{e}}_{3} \times \mathbf{r}\right], \quad b^{\mathrm{Z}}=g \mu_{B} \mathbf{B} \cdot \boldsymbol{\sigma} / 2$, and $b_{\perp}^{\mathrm{Z}}=$ $-g \mu_{B}\left(B_{1} x_{1} / \lambda_{1}+B_{2} x_{2} / \lambda_{2}\right) \sigma_{3} / 4$.

When we apply a Zeeman field, we can treat each induced degeneracy as a two-level system, assuming the spin-orbit matrix elements, $\epsilon_{\mathrm{so}}$, are much less than the single spin mean level spacing, $\Delta=2 \pi \hbar^{2} / m A$, with $m$ the conduction band effective mass and $A$ the dot area. The magnitude of the avoided crossings at the Fermi energy is given by $\epsilon_{\mathrm{so}}=\left|\left\langle\alpha \uparrow\left|\mathcal{H}^{\text {so }}\right| \beta \downarrow\right\rangle\right|$, where $\epsilon_{\alpha}-\epsilon_{\beta}=E_{\mathrm{Z}}$ and $\epsilon_{\alpha}=E_{F}$. We want to find the rms value of $\epsilon_{\mathrm{so}}$. Following Ref. [3], for a closed chaotic dot we may write $\Lambda^{2} \equiv \overline{\left(\epsilon_{\mathrm{so}} / \Delta\right)^{2}}$ as

$$
\Lambda^{2}=\overline{\sum_{\alpha \beta}\left|\left(\mathcal{H}^{\mathrm{so}}\right)_{\alpha \uparrow, \beta \downarrow}\right|^{2} \delta\left(\epsilon_{\alpha}-\epsilon_{\beta}-E_{Z}\right) \delta\left(\epsilon_{\alpha}-E_{F}\right)},
$$

where the overbar indicates ensemble averaging and $O_{a, b} \equiv\langle a|O| b\rangle$.

We rewrite Eq. 3 as in Ref. [3] using the $t$-dependent representation of the delta function and interaction picture operators, and, after summing over $\beta$, find

$$
\Lambda^{2}=\int_{-\infty}^{\infty} \frac{\mathrm{d} t}{\Delta 2 \pi \hbar} e^{-i \omega_{Z} t} \overline{\left\langle\alpha\left|\mathcal{H}_{\mathrm{SF}}^{\mathrm{so}}(t) \mathcal{H}_{\mathrm{SF}}^{\mathrm{so}}{ }^{\dagger}(0)\right| \alpha\right\rangle},
$$

where $\omega_{Z}=E_{\mathrm{Z}} / \hbar$ is the Zeeman frequency, $|\alpha\rangle$ is a typical orbital eigenstate with $\epsilon_{\alpha} \approx E_{\mathrm{F}}$, and $\mathcal{H}_{\mathrm{SF}}^{\mathrm{so}} \equiv$ $\left\langle\uparrow\left|\mathcal{H}^{\text {so }}\right| \downarrow\right\rangle$ is the spin-flip part of $\mathcal{H}^{\text {so }}$. We consider the cubic Dresselhaus term alone, $\mathcal{H}^{c}=\frac{\gamma}{2 \hbar^{3}}\left(p_{2}^{2}-p_{1}^{2}\right)\left(p_{1} \sigma_{2}-\right.$ $\left.p_{2} \sigma_{1}\right)$, and estimate its contribution to $\Lambda$, which we call $\Lambda_{\mathrm{c}}$. For example, if $\mathbf{B}_{\|}=B \hat{\mathbf{e}}_{1}, \mathcal{H}_{\mathrm{SF}}^{c}=\frac{\gamma}{2 \hbar^{3}}\left(p_{2}^{2}-p_{1}^{2}\right) p_{1}$.

We estimate $\Lambda_{\mathrm{c}}$ semiclassically using a billiard model for the quantum dot, where the matrix element in Eq. 4. is replaced by the corresponding expectation value for a classical particle moving at the Fermi velocity, $v_{F}$, starting at a random point moving in a random direction. We consider $B_{\perp}=0$ for these simulations. Each of $2-3 \times 10^{5}$ such trajectories is followed for an equal amount of time, which is generally about 300 bounces total in the forward and backward directions. Increasing the number of trajectories or bounces does not change the results. We calculate $\int \mathrm{d} t e^{-i \omega_{Z}\left(t-t^{\prime}\right)} \mathcal{H}_{\mathrm{SF}}^{\mathrm{c}}(t) \mathcal{H}_{\mathrm{SF}}^{\mathrm{c}}\left(t^{\prime}\right)$ for 100 random initial times $t^{\prime}$ on each trajectory as a function of $\omega_{Z}$, and their average gives $\Lambda_{\mathrm{c}}^{2}$ when multiplied by the appropriate prefactors. We add a damping function to the integrand that sends it smoothly to zero as $t$ approaches the simulation cutoff.

We consider four different billiard shapes and, for specificity, choose parameters corresponding to the
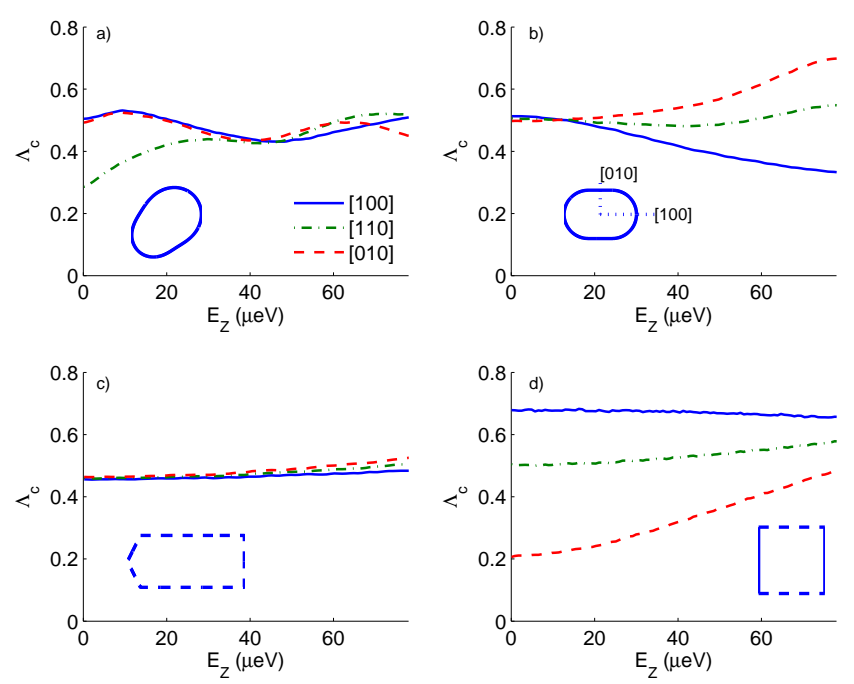

FIG. 1: (color online) Normalized rms avoided crossing due to cubic Dresselhaus spin-orbit coupling, $\Lambda_{\mathrm{c}}$, as a function of Zeeman energy $E_{Z}$ for four billiards with in-plane magnetic field along the indicated directions, with $\gamma=8.5 \mathrm{eV} \AA^{3}[12]$; all billiards have $A=8 \mu \mathrm{m}^{2}$ and $n=5.8 \times 10^{15} \mathrm{~m}^{-2}$. Insets show the billiard shapes with crystal axes. Solid lines indicate specular boundary conditions and dashed lines indicate diffuse boundary conditions. a) has a mixed phase space with small regions of regular trajectories, b) is a stadium billiard, c) is similar to the dot in Ref. [4], with diffuse boundaries to ensure chaos, and d) is a square with diffuse scattering from the top and bottom and specular scattering from the sides (see text).

largest, highest density quantum dot in Ref. [4], with $A=8 \mu \mathrm{m}^{2}$ and $n=5.8 \times 10^{15} \mathrm{~m}^{-2}$. We use $g=0.44$ and $m=0.067 m_{\mathrm{e}}$ where $m_{\mathrm{e}}$ is the electron mass. Fig. 1 (a-c) shows the resulting $\Lambda_{\mathrm{c}}\left(E_{Z}\right)$ for these billiards, for three orientations of $\mathbf{B}_{\|}$, with the Dresselhaus coupling constant chosen to be $\gamma=8.5 \mathrm{eV} \AA^{3}$. For other choices, $\Lambda_{\mathrm{c}}$ scales linearly with $\gamma$. The insets show the shapes of the billiards. For the semiclassical method of estimating $\Lambda_{\mathrm{c}}$ to be valid, we must have $\Delta \ll E_{Z} \ll E_{T}$, where $E_{T}=\hbar v_{F} / \sqrt{A}$ is the Thouless energy. For the case discussed here, $\Delta=0.9 \mu \mathrm{eV}$ and $E_{T} \approx 80 \mu \mathrm{eV}$.

We can understand the approximate scale of $\Lambda_{\mathrm{c}}$ by using a simpler, unphysical billiard. Consider an $L_{x} \times L_{y}$ rectangle with specular reflection boundary conditions on the sides and diffuse boundary conditions on the top and bottom. At each collision with a diffuse wall, we choose the tangential momentum from a uniform distribution on $\left[-p_{F}, p_{F}\right]$ 15]. This choice gives a correct weighting for diffuse scattering and maintains detailed balance. In such a billiard, for $\mathbf{B}_{\|} \| \hat{\mathbf{x}}$, in the limit $E_{Z} \rightarrow 0$,

$$
\left.\Lambda_{\mathrm{c}}^{2}\right|_{E_{Z} \rightarrow 0}=\frac{\gamma^{2}}{\Delta 2 \pi \hbar^{7}}\left\langle\int_{-\infty}^{\infty} \mathrm{d} t p_{y} p_{x}^{2}(t) p_{y} p_{x}^{2}(0)\right\rangle
$$

and we can break each trajectory into segments between collisions with the top/bottom walls. Along each segment, $p_{x}^{2}$ and $p_{y}$ are constant, and the particle takes time 
$t=m L_{y} /\left|p_{y}\right|$ to move from one end of the segment to the other, so we can rewrite Eq. 5 as

$$
\left.\Lambda_{\mathrm{c}}^{2}\right|_{E_{Z} \rightarrow 0}=\frac{\gamma^{2} m L_{y}}{\Delta 2 \pi \hbar^{7}}\left\langle\left|p_{y}(0)\right| p_{x}^{2}(0) \sum_{n=-\infty}^{\infty}(-1)^{n} p_{x, n}^{2}\right\rangle
$$

which we can evaluate explicitly, since the $p_{x, i}^{2}$ are uncorrelated between segments. The particle begins moving in a random direction with $\mathcal{P}\left(p_{x, 0}\right)=\pi^{-1}\left(p_{F}^{2}-p_{x, 0}^{2}\right)^{-1 / 2}$, where $\mathcal{P}$ is the probability density on $\left[-p_{F}, p_{F}\right]$. Since the diffuse boundaries in this billiard are the top and bottom, $\mathcal{P}\left(p_{x, i \neq 0}\right)=1 / 2 p_{F}$. We regularize the infinite sum by $\sum_{n=-\infty}^{\infty}(-1)^{n}=0$, and, noting that $E_{T}=\hbar p_{F} / m L_{y}$, we find $\Lambda_{\mathrm{c}}^{2}=4 \gamma^{2} p_{F}^{6} /\left(45 \pi^{2} \Delta E_{T} \hbar^{6}\right)$. For the parameters in Fig. 1, this gives $\Lambda_{\mathrm{c}}\left(E_{Z} \rightarrow 0\right)=0.678$. Finite values of $E_{Z}$ are not amenable to such simple treatment, but simulations of this billiard appear in Fig. 11d, where the results for $\mathbf{B}_{\|} \| \hat{\mathbf{x}}$, shown by the solid trace, approach the analytic prediction for $E_{Z} \rightarrow 0$.

Avoided crossings have not yet been directly measured in chaotic dots, but our calculations can be related to experiments measuring the conductance $g$ through a quantum dot by Zumbühl et al. [4, 5]. To make this comparison, we need a connection between avoided crossings in a closed dot and properties of the dot with leads attached. Cremers et al., using random matrix theory (RMT), worked out a similar connection for dots with only Rashba and linear Dresselhaus spin-orbit coupling [14]. We point out that the cubic Dresselhaus term can be added easily into the predictions of Cremers et al. without changing their formulas for $\langle g\rangle$ and var $g$ by reinterpreting one of their RMT energy scales to include both linear and cubic spin-orbit terms. We now elaborate.

In Ref. [14], the chaotic quantum dot is connected to two ideal leads with $N \gg 1$ open channels, giving a scattering matrix from the circular orthogonal ensemble. They treat the magnetic field and spin-orbit coupling with a stub model [16] in which the stub has the $M \times M$ perturbation Hamiltonian, $\mathcal{H}_{\mathrm{RMT}}^{\prime}$, given by association to $\mathcal{H}^{\prime}$ in Eq. 2 (without the cubic Dresselhaus term), as

$$
\begin{aligned}
\mathcal{H}_{\mathrm{RMT}}^{\prime}= & \frac{\Delta}{2 \pi}\left[i \mathcal{A}_{0}\left(x \mathbb{1}+a_{\perp} \sigma_{3}\right)+i a_{\|}\left(\mathcal{A}_{1} \sigma_{1}+\mathcal{A}_{2} \sigma_{2}\right)\right. \\
& \left.\left.-\mathbf{b} \cdot \boldsymbol{\sigma}+b_{\perp} \mathcal{B}_{h} \sigma_{3}\right)\right]
\end{aligned}
$$

where $\mathcal{A}_{i}, i=0,1,2$, are real antisymmetric matrices with $\left\langle\operatorname{tr} \mathcal{A}_{i} \mathcal{A}_{j}^{T}\right\rangle=\delta_{i j} M^{2}, \mathcal{B}_{h}$ is a real symmetric matrix with $\left\langle\operatorname{tr} \mathcal{B}_{h}^{2}\right\rangle=M^{2}, M \gg 1$ is the number of channels in the stub, and $x, a_{\perp}, a_{\|}, \mathbf{b}$, and $b_{\perp}$ are dimensionless parameters, with $x$ corresponding to $\mathbf{B}_{\perp}$, $\mathbf{b}$ to the Zeeman field, and $a_{\perp}, a_{\|}$, and $b_{\perp}$ to the similarly named terms in Eq. 2 (without the cubic Dresselhaus term). Dephasing is included by setting $N_{\text {eff }}=N+2 \pi \hbar / \tau_{\phi} \Delta$, where $\tau_{\phi}$ is the dephasing time. Expressions are then obtained for $\langle g\rangle$ and var $g$ as functions of $x, a_{\perp}, a_{\|}, \mathbf{b}, b_{\perp}$, and $N_{\text {eff }}$ to leading order in $1 / N_{\text {eff }}$ [14]. Zumbühl et al. use these results to fit their data.

Without the cubic Dresselhaus term, the correspondence between Eqs. 7 and 2 gives the following mapping from physical parameters to RMT parameters:

$$
\begin{aligned}
x^{2} & =\pi \kappa E_{T} / \Delta\left(\Phi / \Phi_{0}\right)^{2} \quad b=\pi E_{Z} / \Delta \\
a_{\perp}^{2} & =\pi \kappa E_{T} / \Delta\left(A / 4 \lambda_{S O}^{2}\right)^{2} \\
a_{\|}^{2} & =\pi \kappa^{\prime} E_{T} / \Delta\left(A / 4 \lambda_{S O}^{2}\right)^{2}\left(\left(L_{1} / \lambda_{1}\right)^{2}+\left(L_{2} / \lambda_{2}\right)^{2}\right) \\
b_{\perp}^{2} & =\pi \kappa^{\prime \prime}\left(E_{Z}\right)^{2} / E_{T} \Delta\left(A / 4 \lambda_{S O}^{2}\right)
\end{aligned}
$$

where $\Phi$ is the magnetic flux through the quantum dot, $\Phi_{0}=h / 2 e$ is the flux quantum, $\lambda_{S O}=\sqrt{\lambda_{1} \lambda_{2}}, L_{1,2}$ are the linear dimensions of the roughly rectangular dot, oriented along $\hat{\mathbf{e}}_{1,2}$, and $\kappa, \kappa^{\prime}$, and $\kappa^{\prime \prime}$ are geometric factors of order unity [13, 14]. We add the cubic Dresselhaus to this theory by noting that, as a random matrix, the cubic Dresselhaus term in Eq. 2 has the same symmetry as the $a_{\|}$term in Eq. 7 i.e., it contains only $\sigma_{1}$ and $\sigma_{2}$ Pauli matrices. Assuming no correlation with the linear terms, we include the cubic Dresselhaus in $\mathcal{H}_{\mathrm{RMT}}^{\prime}$ by setting $a_{\|}^{2}=a_{\|, 1}^{2}+a_{\|, \mathrm{c}}^{2}$, where $a_{\|, \mathrm{l}}$ is the Rashba and linear Dresselhaus contribution, given by Eq. 8 , and $a_{\|, \mathrm{c}}$ is the cubic Dresselhaus contribution. Since $\mathcal{H}_{\mathrm{RMT}}^{\prime}$ contains the spin-orbit part of the Hamiltonian of the closed quantum dot, we relate $a_{\|, \mathrm{c}}$ to $\Lambda_{\mathrm{c}}$ by finding the rms spin-flip matrix element (with spins quantized along $\mathbf{B}_{\|}$) due to $a_{\|, \mathrm{c}}$, giving $a_{\|, \mathrm{c}}=2 \pi \Lambda_{\mathrm{c}}$. Including the cubic Dresselhaus term in $\mathcal{H}_{\mathrm{RMT}}^{\prime}$ in this way cannot improve any fits to experimental data, as it does not change the fitting equations. However, its inclusion lifts the constraint that $a_{\|} \ll a_{\perp}$ [13], similar to spatially varying spin-orbit strengths [17].

Zumbühl et al. observe weak anti-localization (WAL) in only one of the GaAs/AlGaAs heterostructure quantum dots they study [4, [5], and that dot gives the best defined values of the RMT parameters; we use it for the discussion of our results. The other dots do not contradict this discussion. The dot that displays WAL has area $A=8 \mu \mathrm{m}^{2}$ and electron density $n=5.8 \times 10^{15} \mathrm{~m}^{-2}$, which are the values used in Fig. 1. The $\operatorname{dot}$ has $N=2$ and $N_{\text {eff }}=13.9[4]$.

Zumbühl et al. measure var $g$ as a function of $\mathbf{B}_{\| \text {with }}$ time reversal symmetry broken by a small $B_{\perp}$. They fit to the expression of Cremers et al. [14], with $a_{\|}$(and all parameters except $\kappa^{\prime \prime}$ ) fixed to the value determined from the $\langle g\rangle$ data. We redo the fits to the var $g$ data, constraining only $a_{\|} \geq a_{\|, \mathrm{c}}$, with $a_{\|, \mathrm{c}}$ from our simulations, and $\tau_{\phi}$ fixed to the value determined from $\langle g\rangle$. From Fig. 1] a typical value of $\Lambda_{\mathrm{c}}$ in all our billiard shapes is 0.4 , giving $a_{\|, \mathrm{c}}=2.5$ (8.1) for $\gamma=8.5(27.5) \mathrm{eV} \AA^{3}$ (recalling that $\left.\Lambda_{\mathrm{c}} \propto \gamma\right)$. We find that a value of $a_{\|, \mathrm{c}} \approx 2.5$ is compatible with the experimental data. However, if we require that $a_{\|} \geq 8.1$, the fits to the data become markedly worse.

Zumbühl et al. also measure $\langle g\rangle$ as a function of $B_{\perp}$, which they use to determine $a_{\|}$, finding $a_{\|}=3.1$ [4,,$\underline{5}$, 
18]. Since $a_{\|}^{2}=a_{\|, \mathrm{l}}^{2}+a_{\|, \mathrm{c}}^{2}$, we must have $a_{\|} \geq a_{\|, \mathrm{c}}$, so we conclude that $\gamma=27.5 \mathrm{eVA}^{3}$ is inconsistent with these results, while $\gamma=8.5 \mathrm{eV} \AA^{3}$ is consistent with the data. So both $\langle g\rangle$ and var $g$ data indicate that $\gamma$ should be closer to $9 \mathrm{eV} \AA^{3}$ than $28 \mathrm{eV} \AA^{3}[22]$. Moreover, even with the smaller value of $\gamma$, the cubic Dresselhaus term gives the dominant contribution to $a_{\|}$.

There are only a few other experiments pertaining to the value of $\gamma$ in GaAs. The best, most direct study is the Raman scattering in a GaAs/AlGaAs quantum well by Richards et al. in which they found $\gamma=11.0 \mathrm{eV} \AA^{3}[8]$; the same group also found $\gamma=16.5 \mathrm{eV} \AA^{3}$ in a different sample [9]. A recent experimental value of $\gamma=28 \mathrm{eV} \AA^{3}$ from transport measurements [19] is less direct, includes the cubic Dresselhaus only as a density-dependent renormalization of the linear Dresselhaus, and assumes the Rashba coupling is independent of gate voltage. Theoretical work has indicated that $\gamma$ is smaller in AlGaAs/GaAs heterostructures and superlattices than it is in bulk GaAs [10, 11, 20], so it is possible that experiments are not probing the bulk Dresselhaus coupling, though Ref. 12] predicts $\gamma=8.5 \mathrm{eV} \AA^{3}$ in bulk GaAs. We include as supplementary information a table with experimental and theoretical values of $\gamma$ in GaAs and AlGaAs/GaAs.

Strictly speaking, our calculations are not directly applicable to the $\langle g\rangle$ data of Zumbühl et al., as our calculations assume $E_{Z} \gg \Delta$, and $\langle g\rangle$ is measured with $\mathbf{B}_{\|}=0$. We do not believe, however, that $a_{\|, \mathrm{c}}$ changes significantly as $\mathbf{B}_{\|} \rightarrow 0$; similarly, Cremers et al. consider $a_{\|}$to be constant for all $\mathbf{B}_{\|}[14]$. We believe that $a_{\|, \mathrm{c}}\left(\mathbf{B}_{\|}=0\right)$ can be estimated by simply averaging our results from the different field directions in the limit $\mathbf{B}_{\|} \rightarrow 0$.

Our reinterpretation that $a_{\|}^{2}=a_{\|, 1}^{2}+a_{\|, \mathrm{c}}^{2}$ requires, of course, that $a_{\|, 1}$ be less than 3.1 in the experiment of Zumbühl et al. This reduction of $a_{\|, 1}$ can be absorbed into the geometric parameter $\kappa^{\prime}$ (which was set to 1 without fitting in Zumbühl et al.) without affecting any of the physical parameters, $\tau_{\phi}, \lambda_{S O}$, found by Zumbühl et al. Reducing $\kappa^{\prime}$ is reasonable, as Ref. [14] predicts $\kappa^{\prime}=1 / 3$ for a circular diffusive system.

Since $\Delta \propto A^{-1}$, and in a ballistic system $E_{T} \propto$ $A^{-1 / 2} n^{1 / 2}$, we can see that if the thickness of the 2DEG does not change with density, $a_{\|, 1} \propto A^{7 / 4} n^{1 / 4}$, while $a_{\|, \mathrm{c}} \propto \Lambda_{\mathrm{c}} \propto A^{3 / 4} n^{5 / 4}$. We therefore expect that the cubic Dresselhaus spin-orbit coupling should be relatively more important in small, high density dots, precisely the ones likely to be useful for producing an SFET.

In summary, we have used billiard simulations to estimate the effect of the cubic Dresselhaus term on avoided crossings in a closed chaotic quantum dot. These results are related to the conductance through a dot with ideal leads attached. The cubic Dresselhaus plays a strong and previously ignored role in observed transport properties through quantum dots. Our calculations suggest that 1) the Dresselhaus spin-orbit coupling constant, $\gamma$, in GaAs/AlGaAs heterostructures has a value near 9 $\mathrm{eV} \AA^{3}$ and not the frequently cited value of $27.5 \mathrm{eV} \AA^{3}$, and 2) even with this smaller value of $\gamma$, in the experiments considered the cubic Dresselhaus term provided the bulk of the spin-flip portion of the spin-orbit Hamiltonian, which had previously been assigned to the effects of linear spin-orbit terms. The value of $\gamma$ in this technologically important system deserves further study.

The authors acknowledge a careful reading by Charlie Marcus and helpful conversations with Dominik Zumbühl, Hakan Tureci, Mike Stopa, Emmanuel Rashba, Jeff Miller, Subhaneil Lahiri, Eric Heller, and HansAndreas Engel. The work was supported in part by the Fannie and John Hertz Foundation and NSF grants PHY01-17795 and DMR05-41988.

[1] S. Datta and B. Das, Appl. Phys. Lett. 56, 665 (1990).

[2] J. Schliemann, J. C. Egues, and D. Loss, Phys. Rev. Lett. 90, 146801 (2003).

[3] B. I. Halperin et al., Phys. Rev. Lett. 86, 2106 (2001).

[4] D. M. Zumbühl et al., Phys. Rev. B 72, 081305(R) (2005).

[5] D. M. Zumbühl et al., Phys. Rev. Lett. 89, 276803 (2002).

[6] W. Knap et al., Phys. Rev. B 53, 3912 (1996).

[7] R. Winkler, Spin-Orbit Coupling Effects in TwoDimensional Electon and Hole Systems (Springer, Berlin, 2003).

[8] D. Richards et al., Solid-State Electron. 40, 127 (1996).

[9] B. Jusserand et al., Phys. Rev. B 51, 4707 (1995).

[10] P. V. Santos and M. Cardona, Phys. Rev. Lett. 72, 432 (1994).

[11] P. V. Santos, M. Willatzen, M. Cardona, and A. Cantarero, Phys. Rev. B 51, 5121 (1995).

[12] A. N. Chantis, M. van Schilfgaarde, and T. Kotani, Phys. Rev. Lett. 96, 086405 (2006).

[13] I. L. Aleiner and V. I. Fal'ko, Phys. Rev. Lett. 87, 256801 (2001).

[14] J.-H. Cremers, P. W. Brouwer, and V. I. Fal'ko, Phys. Rev. B 68, 125329 (2003).

[15] M. Fliesser, G. J. O. Schmidt, and H. Spohn, Phys. Rev. E 53, 5690 (1996).

[16] P. W. Brouwer and M. Büttiker, Europhys. Lett. 37, 441 (1997).

[17] P. W. Brouwer, J. N. H. J. Cremers, and B. I. Halperin, Phys. Rev. B 65, 081302(R) (2002).

[18] D. M. Zumbühl, personal communication.

[19] J. B. Miller et al., Phys. Rev. Lett. 90, 076807 (2003).

[20] F. Malcher, G. Lommer, and U. Rössler, Superlattices Microstruct. 2, 267 (1986).

[21] This neglects other spin-flip processes, e.g., hyperfine coupling, which in GaAs is much smaller than spin-orbit effects for quantum dots on the micron scale.

[22] Even using $\Lambda_{\mathrm{c}}=0.2$, the lowest value in Fig. 1, gives $a_{\|, \mathrm{c}}=4.1$ for the larger value of $\gamma$, which is still inconsistent with the experiment. 
TABLE I: Supplementary Information. Magnitude of Dresselhaus spin-orbit coupling constant $\gamma$ in GaAs $\left(e V \AA^{3}\right)$. All results are in bulk GaAs unless otherwise specified.

\begin{tabular}{|c|c|c|}
\hline Ref & $|\gamma|$ & Technique/Comments \\
\hline \multicolumn{3}{|r|}{ EXPERIMENT } \\
\hline$[\mathrm{S} 1]$ & 24.5 & $\begin{array}{l}\text { Optical orientation using Dyakonov-Perel } \\
\text { (DP) spin relaxation time. Conduction } \\
\text { electrons in p-type GaAs. }\end{array}$ \\
\hline$[\mathrm{S} 2]$ & 20.9 & Same as $[\mathrm{S} 1]$ \\
\hline$[\mathrm{S} 3]$ & 26.1 & $\begin{array}{l}\text { GaAs/AlGaAs heterostructure magneto- } \\
\text { conductance measurements. Sets } k_{z}^{2} \rightarrow 0 \\
\text { instead of }\left\langle k_{z}^{2}\right\rangle \text {. }\end{array}$ \\
\hline$[\mathrm{S} 4]$ & 23.5 & $\begin{array}{l}\text { Raman scattering from } 180 \AA \text { GaAs quan- } \\
\text { tum well }(\mathrm{QW}) \text {. Does not include Rashba } \\
\text { term. }\end{array}$ \\
\hline$[\mathrm{S} 5]$ & 34.5 & Same as [S4]. No Rashba term. \\
\hline$[\mathrm{S} 6]$ & $16.5 \pm 3$ & $\begin{array}{l}\text { Raman scattering from GaAs/AlGaAs } \\
\text { heterostructure. }\end{array}$ \\
\hline$[\mathrm{S} 7]$ & 11.0 & $\begin{array}{l}\text { Raman scattering from asymmetric } \\
\text { GaAs/AlGaAs QW. More data than } \\
\text { earlier. }\end{array}$ \\
\hline$[\mathrm{S} 8]$ & $28 \pm 4 ; 31 \pm 3$ & $\begin{array}{l}\text { GaAs/AlGaAs heterostructure magneto- } \\
\text { conductance measurements. }\end{array}$ \\
\hline \multicolumn{3}{|r|}{ THEORY } \\
\hline$[\mathrm{S} 9]$ & $9 ; 8.5$ & $\begin{array}{l}\mathbf{k} \cdot \mathbf{p} \text { theory ; Linear Muffin Tin Orbitals } \\
\text { (LMTO). Room temperature band gap. }\end{array}$ \\
\hline [S10] & $19 ; 30$ & $8 \times 8 ; 14 \times 14 \mathbf{k} \cdot \mathbf{p}$ models. \\
\hline [S11] & 28 & $14 \times 14 \mathbf{k} \cdot \mathbf{p}$ \\
\hline [S12] & $27.57 ; 21.29$ & $\begin{array}{l}\text { k. p. Bulk GaAs ; } \mathrm{Ga}_{.65} \mathrm{Al}_{.35} \mathrm{As} / \mathrm{GaAs} \\
\text { heterostructure. }\end{array}$ \\
\hline [S13] & 14.0 & $\mathbf{k} \cdot \mathbf{p}$ \\
\hline [S14] & $14.9 ; 28.2$ & LMTO ; $\mathbf{k} \cdot \mathbf{p}$ \\
\hline [S15] & 24.12 & 5 level $\mathbf{k} \cdot \mathbf{p}$ \\
\hline [S16] & 19.8 & $\mathbf{k} \cdot \mathbf{p}$ \\
\hline [S17] & 8.9 & $\begin{array}{l}s p_{3} s^{*} \text { tight binding (TB) model of a } \\
\text { GaAs/AlAs superlattice. }\end{array}$ \\
\hline [S18] & 8.5 & TB model of $100 \AA$ GaAs/AlAs QW. \\
\hline [S19] & 24.21 & 5 level $\mathbf{k} \cdot \mathbf{p}$ \\
\hline [S20] & $10 ; 27.5$ & $s p_{3} s^{*}$ TB model $; \mathbf{k} \cdot \mathbf{p}$. \\
\hline [S21] & 27.48 & $\begin{array}{l}14 \text { band } 8 \times 8 \mathbf{k} \cdot \mathbf{p} \text { theory. Notes that value } \\
\text { is reduced to } 19.6 \text { in higher order pertur- } \\
\text { bation theory (p. } 74 \text { ) }\end{array}$ \\
\hline [S22] & 23.6 & TB model used to refine 14 band $8 \times 8 \mathbf{k} \cdot \mathbf{p}$. \\
\hline [S23] & 8.5 & $\begin{array}{l}\text { Quasiparticle self-consistent GW method } \\
\text { (QPscGW) }\end{array}$ \\
\hline
\end{tabular}

[S1] V. A. Marushchak, M. N. Stepanova, and A. N. Titkov, Sov. Phys. Solid State 25, 2035 (1983).

[S2] A. G. Aronov, G. E. Pikus, and A. N. Titkov, Sov. Phys. JETP 57, 680 (1983).

[S3] P. D. Dresselhaus, C. M. A. Papavassiliou, R. G. Wheeler, and R. N. Sacks, Phys. Rev. Lett. 68, 106 (1992).

[S4] D. Richards, B. Jusserand, H. Peric, and B. Etienne, Phys. Rev. B 47, 16028 (1993).

[S5] B. Jusserand, D. Richards, H. Peric, and B. Etienne, Surface Science 305, 247 (1994).

[S6] B. Jusserand, D. Richards, G. Allan, C. Priester, and B. Etienne, Phys. Rev. B 51, 4707 (1995).

[S7] D. Richards, B. Jusserand, G. Allan, C. Priester, and B. Etienne, Solid-State Electron. 40, 127 (1996).

[S8] J. B. Miller, D. M. Zumbühl, C. M. Marcus, Y. B. Lyanda-Geller, D. Goldhaber-Gordon, K. Campman, and A. C. Gossard, Phys. Rev. Lett. 90, 076807 (2003).

[S9] N. E. Christensen and M. Cardona, Solid State Comm. 51, 491 (1984).

[S10] U. Rössler, Solid State Comm. 49, 943 (1984).

[S11] M. Braun and U. Rössler, Journal of Physics C: Solid State Physics 18, 3365 (1985),

[S12] F. Malcher, G. Lommer, and U. Rössler, Superlattices Microstruct. 2, 267 (1986).

[S13] G. E. Pikus, V. A. Marushchak, and A. N. Titkov, Sov. Phys. Semicond. 22, 115 (1988).

[S14] M. Cardona, N. E. Christensen, and G. Fasol, Phys. Rev. B 38, 1806 (1988).

[S15] P. Pfeffer and W. Zawadzki, Phys. Rev. B 41, 1561 (1990).

[S16] H. Mayer and U. Rössler, Solid State Comm. 87, 81 (1993).

[S17] P. V. Santos and M. Cardona, Phys. Rev. Lett. 72, 432 (1994).

[S18] P. V. Santos, M. Willatzen, M. Cardona, and A. Cantarero, Phys. Rev. B 51, 5121 (1995).

[S19] P. Pfeffer and W. Zawadzki, Phys. Rev. B 53, 12813 (1996).

[S20] W. Knap, C. Skierbiszewski, A. Zduniak, E. LitwinStaszewska, D. Bertho, F. Kobbi, J. L. Robert, G. E. Pikus, F. G. Pikus, S. V. Iordanskii, et al., Phys. Rev. B 53, 3912 (1996).

[S21] R. Winkler, Spin-Orbit Coupling Effects in TwoDimensional Electon and Hole Systems (Springer, Berlin, 2003).

[S22] J.-M. Jancu, R. Scholz, E. A. de Andrada e Silva, and G. C. L. Rocca, Physical Review B (Condensed Matter and Materials Physics) 72, 193201 (2005),

[S23] A. N. Chantis, M. van Schilfgaarde, and T. Kotani, Phys. Rev. Lett. 96, 086405 (2006). 